www.nature.com/jhg

\title{
Mutational analysis of GABRG2 in a Japanese cohort with childhood epilepsies
}

\author{
XiuYu Shi ${ }^{1,8}$, Ming-Chih Huang ${ }^{2,3,8}$, Atsushi Ishii ${ }^{1}$, Shuichi Yoshida ${ }^{4}$, Motohiro Okada ${ }^{5}$, Kohtaro Morita ${ }^{6}$, \\ Hiroshi Nagafuji ${ }^{7}$, Sawa Yasumoto ${ }^{1}$, Sunao Kaneko ${ }^{4}$, Toshio Kojima ${ }^{2}$ and Shinichi Hirose ${ }^{1,8}$
}

A few mutations in the gene encoding the gamma 2 subunit of the gamma-aminobutyric acid receptor type A (GABRG2) have been reported in various types of epilepsy. The aim of this study is to investigate the role of GABRG2 in the pathogenesis of childhood epilepsy in a large Japanese cohort. Genetic analysis of GABRG2 was performed on 140 Japanese patients with various childhood epilepsies largely including Dravet syndrome and genetic epilepsy with febrile seizures plus. The mutational analysis identified one novel missense mutation of GABRG2 (c.236A > G: p.N40S) in a patient with generalized tonic-clonic seizures (GTCS). The mutation was heterozygous and replacing a highly conserved Asn residue with a Ser. The affected amino acid was located at residue 40 of the mature GABRG2 protein, which was near the first one of two high-affinity benzodiazepinebinding domains of the $\gamma 2$ subunit (Lys-41-Trp-82). This mutation in such an important position may hamper the function of the channel and contribute to the case's pathogenesis of GTCS.

Journal of Human Genetics (2010) 55, 375-378; doi:10.1038/jhg.2010.47; published online 20 May 2010

Keywords: epilepsy; GABRG2; genetics; mutation

\section{INTRODUCTION}

Epilepsy is a group of heterogeneous disorders characterized by paroxysms resulting from bioelectric hyperexcitation of neuronal networks of the brain. Recently, it has been well recognized that dysfunctions of ion channels expressed in the brain contribute to such hyperexcitation and hence closely associate with the pathogenesis of epilepsy. Accordingly, to date, a number of mutations of the genes encoding ion channels have been identified in various types of epilepsy.

Gamma-aminobutyric acid receptor type $\mathrm{A}\left(\mathrm{GABA}_{\mathrm{A}}\right.$ receptor) is one such ion channel where mutations have been identified in several epilepsy phenotypes. $\mathrm{GABA}_{\mathrm{A}}$ receptor, which is a ligand-gated chloride ion channel, serves as a major component of the neuronal inhibitory system in adult brain. It is considered that majority of the receptors in the brain function as a pentamer consisting of $\alpha 1, \beta 2$ and $\gamma 2$ subunits.

Mutations of the genes encoding the subunits of $\mathrm{GABA}_{\mathrm{A}}$ receptors were so far found in the genes encoding $\alpha 1$ (GABRA1), $\delta$ (GABRD) and $\gamma 2$ subunits $(G A B R G 2) .{ }^{1-4}$ A mutation of GABRA1 was found in autosomal dominant juvenile myoclonic epilepsy, a rare inherited idiopathic epilepsy phenotype. ${ }^{2}$ Several variants of GABRD were not reported as causes of epilepsy but suggested to associate with suscept- ibility to genetic epilepsy with febrile seizures plus (GEFS+). ${ }^{3}$ In contrast, mutations in GABRG2 have been reported as causes of a wide spectrum of epilepsies, from Dravet syndrome to milder conditions such as childhood absence epilepsy, GEFS+ and FS+. ${ }^{1,4-8}$

Although major progress has been made in tying GABRG2 to some categories of epilepsy (that is, GEFS+, childhood absence epilepsy and Dravet syndrome), its role in most kinds of childhood epilepsy such as benign epilepsy of childhood with centrotemporal spikes, generalized tonic-clonic seizures (GTCS), partial epilepsy and so on remains poorly understood. Accordingly, this study further investigates the role of GABRG2 in the pathogenesis of childhood epilepsy.

\section{MATERIALS AND METHODS}

Patients

Our study included 140 pediatric patients who had been diagnosed with epilepsy at various departments of neurology in regional tertiary pediatric hospitals (Table 1). The epilepsy phenotypes included Dravet syndrome, GEFS+, GTCS, partial epilepsy, juvenile myoclonic epilepsy, benign familial neonatal seizures, childhood absence epilepsy, benign epilepsy of childhood with centrotemporal spikes, epilepsy with continuous spikes and waves during slow sleep and progressive myoclonus epilepsy (PME). We also recruited 48 healthy Japanese volunteers as the ethnic matched control group. Each patient

${ }^{1}$ Department of Pediatrics, School of Medicine, Fukuoka University, Fukuoka, Japan; ${ }^{2}$ Computational Systems Biology Research Group, Advanced Science Institute, RIKEN, Yokohama, Japan; ${ }^{3}$ Department of Biological Sciences and Technology, National University of Tainan, Tainan, Taiwan, ROC; ${ }^{4}$ Department of Neuropsychiatory, Hirosaki University Graduate School of Medicine, Hirosaki, Japan; ${ }^{5}$ Department of Psychiatry, Division of Neuroscience, Graduate School of Medicine, Mie University, Mie, Japan; ${ }^{6}$ Department of Neurology, Morita Hospital, Ohgaki, Japan and ${ }^{7}$ Department of Pediatrics, Nagafuji Hospital, Takarazuka, Japan

8These authors contributed equally to this work.

Correspondence: Professor S Hirose, Department of Pediatrics, School of Medicine, Fukuoka University, 45-1 7-chome Nanakuma Jonan-ku, Fukuoka 814-0180, Japan. E-mail: hirose@fukuoka-u.ac.jp

Received 14 December 2009; revised 21 March 2010; accepted 24 March 2010; published online 20 May 2010 
Table 1 Clinical and genetic characteristics of the patients

\begin{tabular}{|c|c|c|c|c|c|c|}
\hline \multirow[b]{2}{*}{ Diagnosis } & \multirow[b]{2}{*}{ Number } & \multicolumn{5}{|c|}{ GABRG2 variants } \\
\hline & & c. $236 A>G$ & c. $315 C>T$ & c. $588 C>T$ & c. $1254 C>T$ & IVS1+18del T \\
\hline GEFS+, FS+ & 36 & 0 & 14 & 27 & 0 & 0 \\
\hline ВECCT & 2 & 0 & 1 & 2 & 0 & 0 \\
\hline BFNC & 7 & 0 & 3 & 5 & 0 & 0 \\
\hline GTCS & 6 & 1 & 3 & 4 & 0 & 0 \\
\hline JME & 9 & 0 & 5 & 9 & 0 & 0 \\
\hline PME & 1 & 0 & 1 & 0 & 0 & 0 \\
\hline PS & 12 & 0 & 6 & 11 & 0 & 0 \\
\hline WEST syndrome & 6 & 0 & 5 & 5 & 0 & 0 \\
\hline Control & 48 & 0 & 22 & 39 & 0 & 0 \\
\hline
\end{tabular}

Abbreviations: BECCT, benign epilepsy of childhood with centrotemporal spikes; BFNC, benign familial neonatal convulsions; CAE, childhood absence epilepsy; CSWS, epilepsy with continuous spikes and waves during slow sleep; FS+, febrile seizures plus; GEFS+, genetic epilepsy with febrile seizures plus; GTCS, generalized tonic-clonic seizures; JME, juvenile myoclonic epilepsy; PME, progressive myoclonus epilepsy; PS, partial epilepsy.

Exon 2 c. $236 \mathrm{~A}>\mathrm{G}(\mathrm{N} 40 \mathrm{~S})$

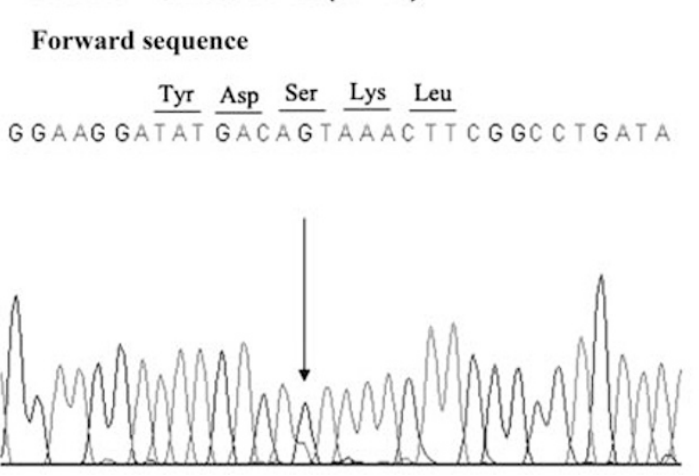

$\begin{array}{ll}\text { Alignment } & \\ \text { Homo sapiens } & \text { LNNLLEGYDNKLRPDIGVKP } \\ \text { Pongo abelii } & \text { LNNLLEGYDNKLRPDIGVKP } \\ \text { Macaca mulatta } & \text { LNNLLEGYDNKLRPDIGVKP } \\ \text { Bos taurus } & \text { LNNLLEGYNKLRPDIGVKP } \\ \text { Equus caballus } & \text { LNNLLEGYDNKLRPDIGVKP } \\ \text { Canis lupus familiaris } & \text { LNNLLEYDNKLRPDIGVKP } \\ \text { Felis catus } & \text { LNNLLEGYDNKLRPDIGVKP } \\ \text { Rattus norvegicus } & \text { LNNLLEGDNKLRPDIGVKP } \\ \text { Mus musculus } & \text { LNNLLEGYDNKLRPDIGVKP } \\ \text { Gallus gallus } & \text { LNGLLEGYNKLRPDIGVKP } \\ \text { Monodelphis domestica } & \text { LNGLLEGYDNKLRPDIGVKP } \\ \text { Danio rerio } & \text { LNSLLDGYDNKLRPDIGVKP }\end{array}$

Figure 1 GABRG2 mutation and alignment of affected amino acid. Arrow indicates where mutation occurs. Rectangular box represents the corresponding amino acid to the amino acid where the mutation occurs, that is highly conserved throughout different species.

or parent/guardian signed an informed consent form approved by the Ethics Review Committee of Fukuoka University or similar committees of the participating institutions.

\section{Genetic analysis}

Using QIAamp DNA Blood kit (Qiagen, Hilden, Germany), genomic DNAs were prepared from ethylenediaminetetraacetic acid-treated whole blood samples. Genetic abnormalities were sought within all 10 exons of GABRG2 and their flanking intronic splice sites by a direct sequencing method with an automatic sequencer as described earlier. ${ }^{9,10}$ Details of the PCR conditions and the primers used are available on request. Reference sequences of mRNA were based on information available from RefSeq, accession numbers: Human GABRG2, NM_198904.

\section{Statistical analysis}

To determine whether the polymorphisms were involved in the pathogenesis of epilepsy, we determined the genotype and allele frequency of the polymorphisms in our patients and in the control population. Data analysis was performed by Fisher's exact test using an SPSS software package (version 13.0). All $P$-values were two-tailed. The significance level was considered to be $5 \%$.

\section{RESULTS}

Mutational analysis for the 140 patients revealed one novel missense mutation (c.236A > G: p.N40S, Figure 1) in one of six patients with GTCS. The mutation was heterozygous and not found in other patients of the cohort or in the 48 ethnic matched control individuals. The DNA of the parents of the patient was not available. The patient was a girl whose first seizure occurred when she was 15-years old, and after that re-occurred once a month. She exhibited the typical seizure and electroencephalographic phenotype and susceptibility to antiepileptic drugs of GTCS supporting that the diagnosis of GTCS. She had no mental retardation or other neurological disorder. Family history of febrile seizures or epilepsy was not found in parents of the patient. The Asn residue at the position of 40, which was replaced with Ser by the mutation, is a highly conserved amino acid throughout many species (Figure 1).

Three exonic variants were found on exon 3 (rs11135176, $n=74$ ), exon 5 (rs211037, $n=105$ ) and exon 10 (c.1254 C/T: p.D379D, $n=3$ ), and one intronic variant (IVS1+18delT, $n=1$ ) was found (Figure 2; Table 1). Variants of rs11135176 and rs211037 were also found in the control population. None of the variants led to any amino-acid changes in the protein sequence. Fisher's exact test showed no 
a

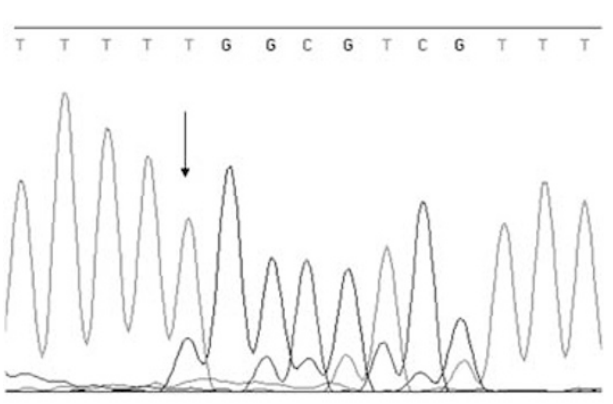

C
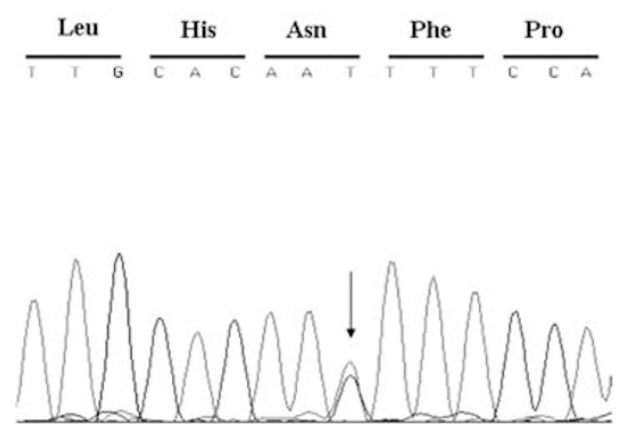

b

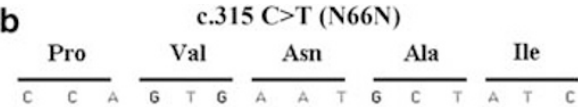

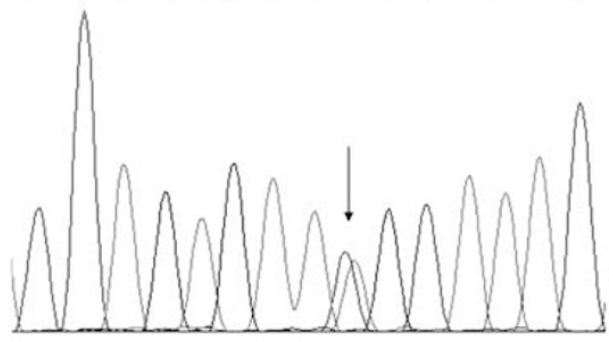

d

c.1254 C > T (D379D)
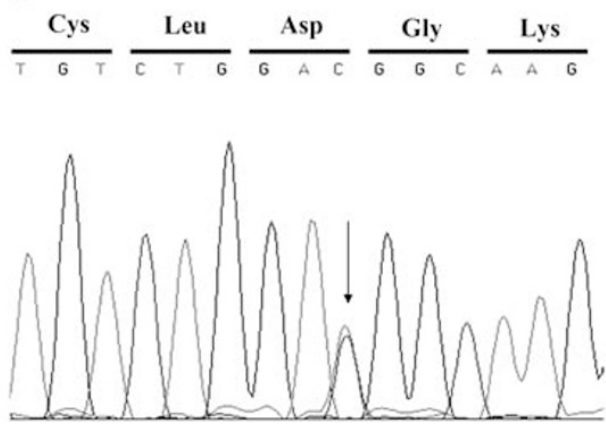

Figure 2 Four GABRG2 variants are identified. Arrows indicate where variants occur. (a) Variant in intron 1. Among these variants there are two known SNPs (b, c) (rs211037 and rs11135176) and one new SNP (d) (c.1254 C>T).

Table 2 Distribution of polymorphisms in the GABRG2 gene in patients and controls

\begin{tabular}{|c|c|c|c|c|c|c|c|c|}
\hline \multirow[b]{2}{*}{ cDNA nucleotide } & \multirow[b]{2}{*}{ Population } & \multicolumn{3}{|c|}{ Genotype count (frequency) } & \multirow[b]{2}{*}{$\mathrm{P}$-value $\mathrm{a}^{\mathrm{a}}$} & \multicolumn{2}{|c|}{ Allele count (frequency) } & \multirow[b]{2}{*}{ P-value ${ }^{b}$} \\
\hline & & $C C$ & $C T$ & $T T$ & & $C$ & $T$ & \\
\hline \multirow[t]{2}{*}{ c. $315 \mathrm{C}>\mathrm{T}$} & Patients & $66(0.47)$ & $61(0.44)$ & $13(0.09)$ & 0.49 & $193(0.69)$ & $87(0.31)$ & 0.30 \\
\hline & Control & $26(0.54)$ & $20(0.42)$ & $2(0.04)$ & & $72(0.75)$ & $24(0.25)$ & \\
\hline \multirow[t]{2}{*}{ c. $588 \mathrm{C}>\mathrm{T}$} & Patients & $35(0.25)$ & $67(0.48)$ & $38(0.27)$ & 0.69 & $137(0.49)$ & $143(0.51)$ & 0.41 \\
\hline & Control & $9(0.19)$ & $24(0.50)$ & $15(0.31)$ & & $42(0.44)$ & $54(0.56)$ & \\
\hline \multirow[t]{2}{*}{ c. $1254 \mathrm{C}>\mathrm{T}$} & Patients & $137(0.98)$ & $3(0.02)$ & $0(0.00)$ & 0.57 & $277(0.99)$ & $3(0.01)$ & 0.57 \\
\hline & Control & $48(1.00)$ & $0(0.00)$ & $0(0.00)$ & & $96(1.00)$ & $0(0.00)$ & \\
\hline
\end{tabular}

aGenotype counts.

${ }^{b}$ Allele counts are compared between patients and control population by Fisher's exact test.

significant differences $(P>0.05)$ in the genotype and allele frequency between the two groups (Table 2).

\section{DISCUSSION}

This study reports one novel missense mutation of GABRG2 (c.236 A $>$ G: p.N40S) in a female with the typical GTCS phenotype. The mutation was heterozygous and not found in 48 ethnic matched control samples. No other relevant genetic variations were found in the 140 patients. To our knowledge, this is the first report of GABRG2 mutation in GTCS providing compelling evidence of the involvement of $G A B R G 2$ in epilepsy.

$\mathrm{GABA}_{\mathrm{A}}$ receptors are the major inhibitory neurotransmitter receptors in the central nervous system, and several anti-epileptic drugs including benzodiazepines, barbiturates and neurosteroids act by enhancing $\mathrm{GABA}_{\mathrm{A}}$ receptor currents. These inhibitory receptors are pentamers formed by assembly of multiple subunit subtypes and the $\alpha 1 \beta 2 \gamma 2$ receptor is the most abundant receptor isoform. The $\gamma 2$ subunit contributes to receptors involved in both phasic and tonic inhibition and underlies the benzodiazepine sensitivity of both modes of inhibition. ${ }^{11}$ It is also critical for receptor trafficking, clustering and synaptic maintenance. ${ }^{12,13} \mathrm{Up}$ to date, at least seven mutations of GABRG2 including missense mutations, nonsense mutations and splice-site mutation have been associated with a broad spectrum of epilepsies. $^{1,4-8,14}$

The N40S mutation identified in this study affects a highly conserved Asn at residue 40 of the mature $\gamma 2$ subunit, thus, the mutation is adjacent to the first one of the two high-affinity benzodiazepine-binding domains of the $\gamma 2$ subunit (Lys-41-Trp-82 in the mature $\gamma 2$ subunit). Wallace et al. ${ }^{15}$ had suggested that R43Q mutation in the benzodiazepine-binding site can attenuate benzodiazepine sensitivity of $\mathrm{GABA}_{\mathrm{A}}$ receptor. Another study on this mutation was shown to accelerate deactivation of the receptor. ${ }^{16}$ These lines of evidence support that the N40S mutation should attenuate the $\mathrm{GABA}_{\mathrm{A}}$ receptor functions whereby increasing the intracortical 
excitability of the brain. This notion accords with the recent findings with the GABRG2 mutations in the N-terminal domain. ${ }^{17}$

In addition, Kang and Macdonald ${ }^{18}$ suggest that with heterozygous expression, the $\mathrm{R} 43 \mathrm{Q}$ mutation may result in impaired receptor trafficking and increased retention of the receptor in intracellular compartments, including the ER. This reduced cell surface expression would result in decreased inhibitory $\mathrm{GABA}_{\mathrm{A}}$ receptor current in vivo, and consequently, an increase in neuronal excitability and epilepsy. Several subsequent studies have identified the retention of mutant receptors in the $\mathrm{ER}^{14}$ and shown that GABRG2 mutations have reduced trafficking either to the membrane surface with relatively normal function ${ }^{18,19}$ or to the surface with impaired function. ${ }^{15,20}$ It is believed that the main electrophysiological deficit of $\mathrm{GABA}_{\mathrm{A}}$ receptor resulting from the mutation is due to intracellular trafficking abnormality of channel molecules. Thus, the N40S mutation may result in an aberrant trafficking of the $\mathrm{GABA}_{\mathrm{A}}$ receptor. We anticipate that this mutation N40S may contribute to the patient's pathogenesis of GTCS.

Recently, Wang et al. ${ }^{21}$ reported that the GABRG2 polymorphism rs211014A allele was higher in the febrile seizures group $(P<0.005)$, suggesting that $G A B R G 2$ polymorphisms may predict susceptibility of febrile seizures. Another study ${ }^{22}$ found that children with the GABRG2 (SNP:rs211037)-C allele had a higher incidence of idiopathic generalized epilepsies, indicating that the GABRG2 (SNP:rs211037)-C allele is a candidate genetic marker for idiopathic generalized epilepsies. In this study, three exonic variants and one intronic variant are identified. Among these variants, c.1254C/T is a novel polymorphism. None of these variants leads to any amino-acid changes in the protein sequence. However, in this study, there are no significant differences for these polymorphisms in genotype and allele frequency between patients and control population, suggesting that they are not involved in the etiology of Japanese childhood epilepsy.

In summary, one novel missense mutation of GABRG2 (N40S) was identified in a patient with GTCS. To our knowledge, there have been no previous reports of mutation of GABRG2 in GTCS. This finding indicates that mutation $G A B R G 2$ can underlie the pathogenesis of GTCS and thus reinforces the involvement of $\mathrm{GABA}_{\mathrm{A}}$ receptors in epilepsy. However, the contribution of mutations of GABRG2 per se to the pathogenesis of epilepsy remains unclear because we do not have sufficient patients. Further studies will involve gathering more cases especially GTCS, progressive myoclonus epilepsy patients and performing the $G A B R G 2$ analysis.

\section{ACKNOWLEDGEMENTS}

We are indebted to all members of the family for their helpful cooperation in this study. We thank Ms Takako Umemoto and Hideko Takeda for formatting and typing the manuscript and Ms Minako Yonetani and Akiyo Hamachi for the technical assistance. This study was supported in part by Grants-in-Aid for Scientific Research (S) 16109006, (A) 18209035 and 21249062, Exploratory Research 1659272, and 'High-Tech Research Center' Project for Private Universities-matching fund subsidy from the Ministry of Education, Culture, Sports, Science and Technology, 2006-2010 'The Research Center for the Molecular Pathomechanisms of Epilepsy, Fukuoka University', Research Grants (19A-6) and (21B-5) for Nervous and Mental Disorders from the Ministry of Health, Labor and Welfare and the Central Research Institute of Fukuoka University and an in-house budget of RIKEN.
1 Baulac, S., Huberfeld, G., Gourfinkel-An, I., Mitropoulou, G., Beranger, A., Prud'homme, J. F., et al. First genetic evidence of $G A B A(A)$ receptor dysfunction in epilepsy: a mutation in the gamma2-subunit gene. Nat. Genet. 28, 46-48 (2001).

2 Cossette, P., Liu, L., Brisebois, K., Dong, H., Lortie, A., Vanasse, M. et al. Mutation of GABRA1 in an autosomal dominant form of juvenile myoclonic epilepsy. Nat. Genet. 31, 184-189 (2002)

3 Dibbens, L. M., Feng, H. J., Richards, M. C., Harkin, L. A., Hodgson, B. L., Scott, D. et al. GABRD encoding a protein for extra- or peri-synaptic GABAA receptors is a susceptibility locus for generalized epilepsies. Hum. Mol. Genet. 13, 1315-1319 (2004).

4 Harkin, L. A., Bowser, D. N., Dibbens, L. M., Singh, R., Phillips, F., Wallace, R. H. et al. Truncation of the GABA(A)-receptor gamma2 subunit in a family with generalized epilepsy with febrile seizures plus. Am. J. Hum. Genet. 70, 530-536 (2002).

5 Audenaert, D., Schwartz, E., Claeys, K. G., Claes, L., Deprez, L., Suls, A., et al. A novel GABRG2 mutation associated with febrile seizures. Neurology 67, 687-690 (2006).

6 Kananura, C., Haug, K., Sander, T., Runge, U., Gu, W., Hallmann, K. et al. A splice-site mutation in GABRG2 associated with childhood absence epilepsy and febrile convulsions. Arch. Neurol. 59, 1137-1141 (2002).

7 Sun, H., Zhang, Y., Liang, J., Liu, X., Ma, X., Wu, H. et al. SCN1A, SCN1B, and GABRG2 gene mutation analysis in Chinese families with generalized epilepsy with febrile seizures plus. J. Hum. Genet. 53, 769-774 (2008).

8 Wallace, R. H., Marini, C., Petrou, S., Harkin, L. A., Bowser, D. N., Panchal, R. G. et al. Mutant GABA(A) receptor gamma2-subunit in childhood absence epilepsy and febrile seizures. Nat. Genet. 28, 49-52 (2001).

9 Wang, J. W., Kurahashi, H., Ishii, A., Kojima, T., Ohfu, M., Inoue, T. et al. Microchromosomal deletions involving SCN1A and adjacent genes in severe myoclonic epilepsy in infancy. Epilepsia 49, 1528-1534 (2008).

10 Fukuma, G., Oguni, H., Shirasaka, Y., Watanabe, K., Miyajima, T., Yasumoto, S. et al. Mutations of neuronal voltage-gated $\mathrm{Na}+$ channel alpha 1 subunit gene SCN1A in core severe myoclonic epilepsy in infancy (SMEI) and in borderline SMEI (SMEB). Epilepsia 45, 140-148 (2004).

11 Prenosil, G. A Schneider Gasser, E.M. Rudolph, U Keist, R Fritschy, J. M \& Vogt, K. E. Specific subtypes of GABAA receptors mediate phasic and tonic forms of inhibition in hippocampal pyramidal neurons. J. Neurophysiol. 96, 846-857 (2006).

12 Essrich, C., Lorez, M., Benson, J. A., Fritschy, J. M. \& Luscher, B. Postsynaptic clustering of major GABAA receptor subtypes requires the gamma 2 subunit and gephyrin. Nat. Neurosci. 1, 563-571 (1998).

13 Schweizer, C., Balsiger, S., Bluethmann, H., Mansuy, I. M., Fritschy, J. M., Mohler, H. et al. The gamma 2 subunit of $\operatorname{GABA}(\mathrm{A})$ receptors is required for maintenance of receptors at mature synapses. Mol. Cell Neurosci. 24, 442-450 (2003).

14 Hirose, S. A new paradigm of channelopathy in epilepsy syndromes: intracellular trafficking abnormality of channel molecules. Epilepsy Res. 70 (Suppl 1), S206S217 (2006).

15 Bianchi, M. T., Song, L., Zhang, H. \& Macdonald, R. L. Two different mechanisms of disinhibition produced by GABAA receptor mutations linked to epilepsy in humans. J. Neurosci. 22, 5321-5327 (2002).

16 Bowser, D. N., Wagner, D. A., Czajkowski, C., Cromer, B. A., Parker, M. W., Wallace, R. $\mathrm{H}$. et al. Altered kinetics and benzodiazepine sensitivity of a GABAA receptor subunit mutation [gamma 2(R43Q)] found in human epilepsy. Proc. Natl Acad. Sci. USA 99, 15170-15175 (2002).

17 Fedi, M., Berkovic, S. F., Macdonell, R. A., Curatolo, J. M., Marini, C. \& Reutens, D. C. Intracortical hyperexcitability in humans with a GABAA receptor mutation. Cereb. Cortex 18, 664-669 (2008).

18 Kang, J. Q. \& Macdonald, R. L. The GABAA receptor gamma2 subunit R43Q mutation linked to childhood absence epilepsy and febrile seizures causes retention of alpha1beta2gamma2S receptors in the endoplasmic reticulum. J. Neurosci. 24, 8672-8677 (2004).

19 Macdonald, R. L., Kang, J. Q., Gallagher, M. J. \& Feng, H. J. GABA(A) receptor mutations associated with generalized epilepsies. Adv. Pharmacol. 54, 147-169 (2006).

20 Feng, H. J., Kang, J. Q., Song, L., Dibbens, L., Mulley, J. \& Macdonald, R. L. Delta subunit susceptibility variants E177A and $\mathrm{R} 22 \mathrm{OH}$ associated with complex epilepsy alter channel gating and surface expression of alpha4beta2delta GABAA receptors. J. Neurosci. 26, 1499-1506 (2006).

21 Xiumin, W., Meichun, X. \& Lizhong, D. Association analysis of gamma2 subunit of gamma-aminobutyric acid (GABA) type A receptor and voltage-gated sodium channel type II alpha-polypeptide gene mutation in southern Chinese children with febrile seizures. J. Child Neurol. 22, 714-719 (2007).

22 Chou, I. C., Lee, C. C., Tsai, C. H., Tsai, Y., Wan, L., Hsu, Y. A. et al. Association of GABRG2 polymorphisms with idiopathic generalized epilepsy. Pediatr. Neurol. 36, 4044 (2007). 